\title{
ERRATUM
}

\section{Erratum to: Parity induced edge-current saturation and current distribution in zigzag-edged graphene nano-ribbon devices}

\author{
Satofumi Souma • Matsuto Ogawa • \\ Takahiro Yamamoto • Kazuyuki Watanabe
}

Published online: 22 April 2011

(C) Springer Science+Business Media LLC 2011

\section{Erratum to: Int J Comput Electron DOI 10.1007/s10825-011-0352-0}

In the original version of this article, Fig. 13 appears incorrectly. The correct version of the figure is given in the right column.
The online version of the original article can be found under doi:10.1007/s10825-011-0352-0.

S. Souma $(\bowtie) \cdot$ M. Ogawa

Department of Electric and Electronic Engineering, Kobe University, 1-1 Rokkodai, Nada, Kobe 657-8501, Japan e-mail: ssouma@harbor.kobe-u.ac.jp

T. Yamamoto

Department of Materials Engineering, The University of Tokyo,

Tokyo, Japan

K. Watanabe

Department of Physics, Faculty of Science, Tokyo University

of Science, 1-3 Kagurazaka, Shinjuku-ku, Tokyo 162-8601, Japan

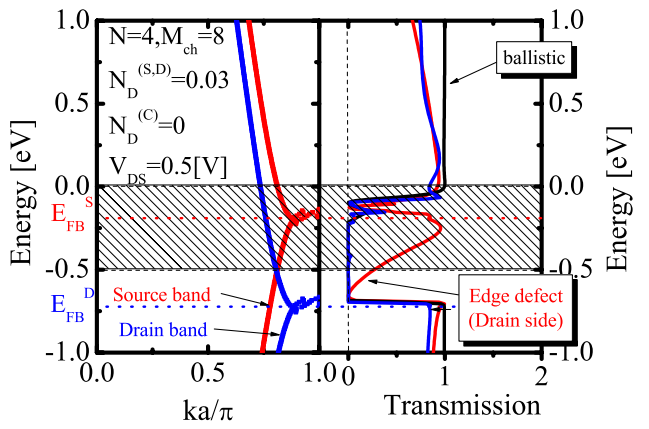

Fig. 13 Electronic band structures in the source and the drain electrodes (left) and the transmission probabilities (right) of ZGNR $n-i-n$ structure with $N=4$ and $M_{\mathrm{ch}}=8$ where the source drain bias is $V_{\mathrm{DS}}=0.5[\mathrm{~V}]$, and the doping level in the electrode and the channel are $N_{\mathrm{D}}^{\mathrm{S}, \mathrm{D}}=0.03$ and $N_{\mathrm{D}}^{\mathrm{C}}=0$ per atom, respectively. Results for the ballistic case and the defective case (single edge vacancy near the drain electrode) are compared. Shaded energy range corresponds to the bias window that contributes to the current 